PAPER

\title{
Risk factors for intracardiac thrombus in patients with recent ischaemic cerebrovascular events
}

\author{
S Sen, S Laowatana, J Lima, S M Oppenheimer
}

See Editorial Commentary, p 1373

J Neurol Neurosurg Psychiatry 2004;75:1421-1425. doi: 10.1136/jnnp.2004.038687

See end of article for authors' affiliations

.....................

Correspondence to: Dr Stephen M

Oppenheimer, 101

Wendover Road,

Baltimore, MD 21218 ,

USA; soppenh@hotmail.

com

Received 5 February 2004

Revised 16 April 2004

Accepted 2 May 2004
Background: Intracardiac thrombus is a common cause of cardiogenic cerebral ischaemia. Stroke recurrence is high, but thrombus detection with therapeutic intervention can reduce the risk. Accurate detection requires transoesophageal echocardiography (TOE), which is semi-invasive and costly.

Objective: To identify risk factors for cardiac thrombus, enabling selection of patients for TOE and initiation of measures to prevent the formation of cardiac thrombus.

Methods: 151 consecutive patients with ischaemic stroke or transient ischaemic attacks (mean age 62 years) underwent TOE for intracardiac thrombus detection within one week of a qualifying event.

Results: Intracardiac thrombus was found in $26 \%$ of the patients (70\% in the left atrial appendage). Multivariate analysis indicated the following clinical correlates: large stroke, odds ratio $(O R)=2.8(95 \%$ confidence interval, 1.2 to 6.4); symptomatic coronary artery disease, $\mathrm{OR}=3.0(1.2$ to 7.4 ); and ECG evidence of ischaemia, $\mathrm{OR}=2.8$ (1.1 to 7.7). Neither carotid stenosis $>70 \%$, nor stroke location correlated with the presence of thrombus.

Conclusions: Clinical factors correlate with and appear to be risk factors for cardiac thrombus in patients with recent cerebral ischaemia. These may be used to select appropriate patients for invasive and costly TOE investigation, irrespective of the presence of significant carotid stenosis ( $\geqslant 70 \%$ ) or stroke location.
C linical stroke registries show that $20-40 \%$ of strokes are caused by cardiogenic emboli. ${ }^{12}$ These carry a signifiaetiology ${ }^{3}$ and also a higher recurrence risk. ${ }^{4}$ Intracardiac thrombus is a common cardiac abnormality with a high potential for embolisation to the cerebral vasculature. ${ }^{3}$ It represents a definite indication for long term anticoagulation to reduce the threat of recurrent stroke ${ }^{3}$ and possibly to dissolve the thrombus. ${ }^{5}$

Transoesophageal echocardiography (TOE) has greatly improved intracardiac thrombus detection, as thrombi are usually located in the left atrium and left atrial appendageregions that are particularly well visualised, ${ }^{67}$ with a sensitivity of $100 \%$ and a specificity of $99 \% .^{8}$ TOE is superior to transthoracic echocardiography (TTE) for imaging the left atrium and the left atrial appendage. ${ }^{6-11}$ However, concerns have been raised about the cost-effectiveness and invasiveness of TOE. ${ }^{12}$

A hypercoagulable state may contribute to the aetiology of cardiac thrombus. Patients with TOE findings of spontaneous echocardiographic contrast (a marker of thromboembolism) are in a relative hypercoagulable state. ${ }^{13}$ To our knowledge a correlation between a hypercoagulable state and intracardiac thrombus has not been investigated.

Our primary objective was to investigate whether the presence of cardiac thrombus correlates with common clinical variables after cerebral ischaemia, thereby refining selection criteria for TOE. The secondary objective was to investigate whether haematological or hypercoagulation abnormalities are associated with intracardiac thrombus.

\section{METHODS}

In all, 164 consecutive patients satisfied the following entry criteria:

- ability of non-comatose patient or surrogate to provide informed consent;

- age more than 18 years;
- first symptomatic ischaemic stroke or transient ischaemic attack (TIA), with onset within the previous seven days.

All enrolled patients underwent stroke risk factor assessment, ECG, and 24 hour Holter examination, haematological assessment, and brain computed tomography (CT) or magnetic resonance imaging (MRI). Carotid artery patency was assessed in $87 \%$ of the cases, either by carotid duplex ultrasound investigation or by magnetic resonance angiography (MRA). Patients without symptomatic coronary artery disease underwent adenosine thallium myocardial perfusion scanning to assess whether they had asymptomatic coronary artery disease. The study was approved by the joint committee on clinical investigation of Johns Hopkins Hospital.

Multiplanar TOE was carried out in 151 of the 164 patients within seven days of ischaemic stroke or TIA. Of the remainder, there were nine refusals and TOE could not be done on the initial admission in four. Two cardiologists blinded to the patients' clinical data reviewed examination videotapes independently. In cases of discrepancy, results were adjudicated independently by a third cardiologist. Discrepancies in left ventricular thrombus status were clarified by cross sectional echocardiography.

Symptomatic coronary artery disease was defined as a confirmed history of myocardial infarction, angina, or ischaemic cardiac failure. Stroke location was classified using the Oxfordshire health study criteria. ${ }^{14}$ Large artery atherosclerotic source of thrombosis or embolism was assessed in $87 \%$ of patients by carotid ultrasound or MRA. A routine PA $x$ ray view of the chest was used to assess cardiomegaly. A cardiothoracic ratio of $\geqslant 0.5$ was considered significant. ST elevation or depression of $\geqslant 1.5 \mathrm{~mm}$ with or without $\mathrm{T}$ wave flattening, $\mathrm{T}$ wave inversion, or pathological $\mathrm{Q}$ wave on

Abbreviations: OCSP, Oxford Community Stroke Project; TOAST, The Obesity Awareness and Solutions Trust; TOE, transoesophageal echocardiography; TTE, transthoracic echocardiography 
$111(74 \%)$ No thrombus

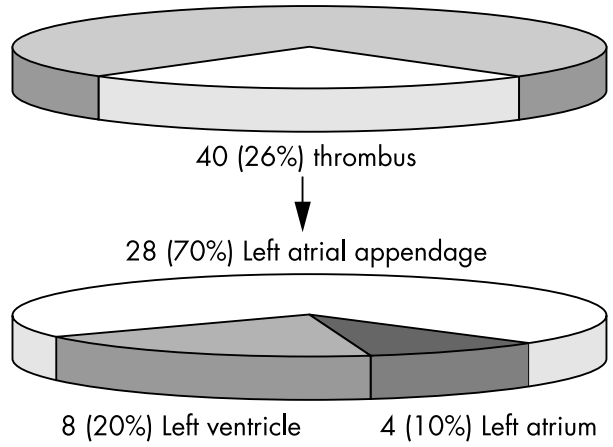

Figure 1 Incidence and distribution of intracardiac thrombus detected by transoesophageal echocardiography in patients with recent strokes or transient ischaemic attacks.

routine 12-lead ECG at the time of admission were considered signs of cardiac ischaemia. Paroxysmal or continuous atrial fibrillation was documented on 24 hour Holter examination done within seven days of stroke or TIA symptom onset.

All blood tests were carried out within seven days of cerebral ischaemia onset. All patients not anticoagulated (oral or intravenous) were assessed for prothrombin time (PT), activated partial thromboplastin time (aPTT), thrombin time, diluted Russell viper venom test (dRVVT), protein C, protein S, and antithrombin III activity. Sixty five patients had assessments of activated protein $\mathrm{C}$ resistance, and 111 had assessment of anticardiolipin antibody activity (IgG, IgA, and IgM classes). Patients without renal failure (plasma creatinine $\leqslant 220 \mu \mathrm{mol} / \mathrm{l}$ ) had measurement of fasting blood homocysteine.

The association of stroke subtype with the presence of intracardiac thrombus was explored using Fisher's exact test. Two tailed $t$ tests compared mean ages of patients with and without intracardiac thrombus. Univariate analysis included Fisher's exact test and estimation of odds ratios for each stroke factor other than age. Finally, multiple logistic regression analyses were carried out to obtain the odds ratio (OR) adjusted for all other measured risk factors.

Statistical analyses were done using Instat version 2.0 (GraphPad, San Diego, California, USA) (two tailed $t$ test, Fisher's exact test, and univariate odds ratio analysis) and SPSS for Windows, version 10.0 (SPSS, Chicago, Illinois, USA) (multivariate analysis and $\kappa$ analysis).

\section{RESULTS}

Intracardiac thrombus was identified in $26 \%$ of ischaemic stroke/TIA patients (70\% located in the left atrial appendage, fig 1) with a $\kappa$ index of $0.91 \quad(p<0.0001)$, indicating good interobserver reliability. No demographic or risk factors differentiated between those patients with and without intracardiac thrombus (table 1). The majority of patients with intracardiac thrombus (55\%) had had a large stroke $(\mathrm{p}=0.025)$ (table 2). There was no difference in clinical stroke presentation between the 38 patients with thrombus (five with total anterior and 15 with partial anterior circulation infarction (TACI, PACI), 14 with lacunar infarction (LACI), and four with posterior circulation infarction (POCI)) and the 88 patients without thrombus (seven with TACI, 35 with PACI, 36 with LACI, and 10 with POCI). Nearly $25 \%$ of patients with small vessel disease in this study, however, were found to have an intracardiac thrombus (table 2). The majority of TIA patients (92\%) lacked echocardiographic evidence of intracardiac thrombus $(p=0.025)$. All haematological measurements were taken
Table 1 Demographics and risk factors of 151 patients with stroke or transient ischaemic attack who underwent transoesophageal echocardiographic assessment for intracardiac thrombus

\begin{tabular}{llll}
\hline Risk factor & $\begin{array}{l}\text { Thrombus } \\
\mathbf{n = 4 0}\end{array}$ & $\begin{array}{l}\text { No thrombus } \\
\mathbf{n = 1 1 1}\end{array}$ & $\mathbf{p ~ V a l u { } ^ { * }}$ \\
\hline Age (years) (mean (SD)) & $63(12.8)$ & $62(12.7)$ & 0.7 \\
Male sex & $17(42 \%)$ & $56(50 \%)$ & 0.46 \\
Black race & $28(70 \%)$ & $70(63 \%)$ & 0.56 \\
Hypertension & $30(75 \%)$ & $82(74 \%)$ & 1.0 \\
Diabetes & $10(25 \%)$ & $34(31 \%)$ & 0.55 \\
Hypercholesterolaemia & $15(37 \%)$ & $44(40 \%)$ & 0.85 \\
Smoking & $25(62 \%)$ & $71(64 \%)$ & 1.0 \\
Alcohol use & $15(37 \%)$ & $38(34 \%)$ & 0.7 \\
Carotid stenosis $\geqslant 70 \%$ & $4 / 35(11 \%)$ & $23 / 96(24 \%)$ & 0.15 \\
PVD & $6(15 \%)$ & $23(21 \%)$ & 0.49 \\
History of CAD & $17(42 \%)$ & $30(27 \%)$ & 0.08 \\
Atrial fibrillation & $11(27 \%)$ & $15(14 \%)$ & 0.04 \\
\hline
\end{tabular}

*Two tailed $t$ test or Fisher's exact test.

$C A D$, coronary artery disease; PVD, peripheral vascular disease.

at a median of five days from symptom onset in both thrombus and non-thrombus patients. No haematological variable or coagulation profile predicted the presence or absence of intracardiac thrombus (tables 3 and 4).

Univariate testing (table 1, fig 2) suggests that, of the common vascular risk factors, only coronary artery disease might indicate the presence of intracardiac thrombus (univariate odds ratio analysis, $\mathrm{OR}=2.1$ (95\% confidence interval, 1.0 to 4.4$) ; \mathrm{p}=0.08$ ). On multivariate analysis, the adjusted OR for coronary artery disease became highly significant $(\mathrm{OR}=3.0 \quad(1.2$ to 7.4$) ; \mathrm{p}=0.017) \quad$ (fig 2). The relation between radiological large stroke and intracardiac thrombus was evident on univariate odds ratio analysis $(\mathrm{OR}=2.2(1.1$ to 4.7$))$, persisting on multivariate analysis (adjusted OR $=2.8$ (1.2 to 6.4)) (fig 2).

Of the cardiac variables, univariate analysis suggested that only atrial fibrillation on ECG/Holter $(\mathrm{OR}=2.7$ ( 1.1 to 6.5$)$ ) and left ventricular systolic dysfunction manifested by wall motion abnormality on TOE $(\mathrm{OR}=3.4(1.7$ to 7.1$))$ were correlated with intracardiac thrombus (fig 3). Multivariate analysis showed that ischaemia on ECG (adjusted OR $=2.8$ (1.1 to 7.7) and systolic left ventricular dysfunction (adjusted $\mathrm{OR}=3.9$, 95\% CI 1.5-10.5) were correlated with detection of intracardiac thrombus on TOE.

\section{DISCUSSION}

In this prospective study, $70 \%$ of all thrombi were located in the left atrial appendage. Using multivariate analysis, clinical predictors independently associated with thrombus detection included radiological large stroke, coronary artery disease, and ECG evidence of ischaemia. On TOE, left ventricular systolic dysfunction was associated with the presence of a thrombus. Using the OCSP criteria, intracardiac thrombus was not associated with any stroke subtype, nor did the presence of significant carotid stenosis exclude this finding. No haematological or coagulation variable significantly

Table 2 Clinical neuroradiological classification of ischaemic stroke/TIA

\begin{tabular}{llll}
\hline Type & $\begin{array}{l}\text { Thrombus } \\
(\mathbf{n}=\mathbf{4 0})\end{array}$ & $\begin{array}{l}\text { No thrombus } \\
(\mathbf{n}=\mathbf{1 1} 1)\end{array}$ & $\mathbf{p ~ V a l u \mathbf { 1 } ^ { * }}$ \\
\hline Large vessel & 22 & 38 & 0.025 \\
Small vessel & 16 & 50 & 0.71 \\
TIA & 2 & 23 & 0.025 \\
\hline
\end{tabular}

*Fisher's exact test.

TIA, transient ischaemic attack. 
Table 3 Haematological profile of patients with stroke/TIA with and without intracardiac thrombus

\begin{tabular}{|c|c|c|c|}
\hline Variable & Thrombus $(n=40)$ & No thrombus $(n=111)$ & p Value* \\
\hline Haemoglobin (g/dl) & $12.5(1.7)$ & $12.9(1.8)$ & 0.2 \\
\hline Packed cell volume (\%) & $37.4(5.0)$ & $38.4(5.2)$ & 0.3 \\
\hline Platelet count $\left(\mathrm{k} / \mathrm{mm}^{3}\right)$ & $271.1(89)$ & $263.1(81)$ & 0.6 \\
\hline WBC count $\left(/ \mathrm{mm}^{3}\right)$ & 8611 (2572) & 7708 (2603) & 0.06 \\
\hline 25th centile & 6375 & 6000 & \\
\hline 50th centile & 8650 & 7600 & \\
\hline 75th centile & 10500 & 9100 & \\
\hline $\operatorname{ESR}(\mathrm{mm} / \mathrm{h})$ & $44(29)(n=33)$ & $42(29)(n=77)$ & 0.8 \\
\hline Plasma viscosity (plas/sol) & $1.89(0.23)(n=25)$ & $1.81(0.17)(n=62)$ & 0.1 \\
\hline
\end{tabular}

correlated with the presence of intracardiac thrombus in this study.

Cardiac thrombus was detected in $26 \%$ of patients with acute cerebral ischaemia, whereas previous TOE investigations enrolling smaller patient numbers have reported a lower incidence of 3-22\%.6 ${ }^{61011} 15$ In this study, a more accurate multiplanar TOE technique was used in all patients, as opposed to monoplanar, ${ }^{7}$ biplanar, ${ }^{10}{ }^{11}$ or both monoplanar and biplanar TOE. ${ }^{6}{ }^{15}$ Likewise, the sensitivity of detection of intracardiac thrombus might have been heightened by a systematic approach of investigating the left atrium, left atrial appendage, and left ventricle for the presence of thrombus in all stroke patients as part of the protocol, without selection for age or lack of stroke aetiology. Indeed, when stratified by age, the incidence of thrombus detection in patients aged under 65 years was 9\% (seven of 79) in keeping with previously reported data. When the age cut off is increased to 75 years (an age group often excluded from TOE examination), the corresponding figure is $25 \%$ (31 of 122). Patients were not excluded because of stroke subtype (for example, lacunar strokes) or TIA.

As in the present study, previous investigations have confirmed that the left atrial appendage is the commonest site for intracardiac thrombus. ${ }^{6} 71516$ This may reflect the lower blood flow velocity in the appendage than in the rest of the left heart. ${ }^{16}$

Among clinical variables, intracardiac thrombus correlated with coronary artery disease. This could be related to the ventricular wall motion abnormalities observed in patients with coronary artery disease with or without ischaemic cardiomyopathy. ${ }^{17-19}$ In this study, left ventricular dysfunction was an independent predictor of intracardiac thrombus on multivariate analysis, in keeping with this suggestion. An additional causal relation probably reflects the higher incidence of atrial fibrillation in patients with coronary artery disease $(52 \%$ in this study) than those without $(27 \%$, $\mathrm{p}=0.005)$.

Intracardiac thrombus also correlated with large strokes on CT/MRI-in agreement with recent studies showing that patients with cardiogenic brain emboli have larger strokes with worse prognosis than those without. ${ }^{3}$ This may also be related to a significantly higher incidence of atrial fibrillation in the thrombus group $(27 \%)$ compared with the nonthrombus group $(13 \%)(p=0.045)$. This indicates a possible need for TOE examination in patients with stroke and atrial fibrillation, even if they are to be anticoagulated, as this combination may place patients at higher risk of stroke recurrence and indicates a possible requirement for more intense anticoagulation. Previous studies have reported that patients with atrial fibrillation have larger and more severe strokes, which has been attributed to cerebral blood flow reduction in the presence of this arrhythmia. ${ }^{20}$ The association between atrial fibrillation and intracardiac thrombus disappeared on multivariate analysis, when only ECG evidence of ischaemia was predictive. This could reflect a causative association between cardiac ischaemia and atrial fibrillation.

In this study, $28 \%$ of patients with small vessel occlusive disease, as defined by TOAST criteria, had TOE evidence of intracardiac thrombus. Traditionally, small vessel occlusive disease is attributed to hyaline arteriosclerosis of small arterioles mediated by hypertension and diabetes, and such patients are not pursued with TOE investigations. In a recent biplanar TOE study of 22 patients with lacunar stroke no left

\begin{tabular}{|c|c|c|c|}
\hline Test (normal value) & Thrombus $(n=40)$ & No thrombus $(n=111)$ & $p$ Value* \\
\hline PT (11.5-13.5 s) & $12.4(0.7)(n=22)$ & $12.2(0.7)(n=87)$ & 0.2 \\
\hline aPTा (20.0-31.6 s) & $26.7(4.1)(n=22)$ & $25.4(4.8)(n=87)$ & 0.2 \\
\hline dRVVT $(29.3-42.8 \mathrm{~s})$ & $37.5(15)(n=17)$ & $34.2(9.3)(n=52)$ & 0.3 \\
\hline Fibrinogen $(150-400 \mathrm{mg} / \mathrm{dl})$ & $499(206)(n=37)$ & $457(142)(n=85)$ & 0.2 \\
\hline Protein C deficiency (70-180\%) & $2 / 19(10 \%)$ & $2 / 62(3 \%)$ & 0.2 \\
\hline Protein S deficiency (65-140\%) & $0 / 19(0)$ & $3 / 62(5 \%)$ & 1.0 \\
\hline AT III deficiency (80-120\%) & $4 / 19(21 \%)$ & $6 / 62(10 \%)$ & 0.2 \\
\hline APC resistance $(>2.1)$ & $2 / 14(14 \%)$ & $5 / 51(10 \%)$ & 0.6 \\
\hline ACA $\lg G(<10 \mathrm{gpl} / \mathrm{ml})$ & $1 / 35(3 \%)$ & $1 / 76(1 \%)$ & 1.0 \\
\hline ACA IgA $(<10$ sdu $)$ & $1 / 35(3 \%)$ & $3 / 76(4 \%)$ & 1.0 \\
\hline ACA $\lg M(<10 \mathrm{mpl} / \mathrm{ml})$ & $2 / 35(6 \%)$ & $1 / 76(1 \%)$ & 0.2 \\
\hline Hyperhomocysteinaemia $(5.1-13.9 \mu \mathrm{mol} / \mathrm{l})$ & $2 / 18(11 \%)$ & $8 / 51(16 \%)$ & 1.0 \\
\hline
\end{tabular}

Values are mean (SD) or $\mathrm{n}(\%)$.

${ }^{*}$ T Test or Fisher's exact test.

ACA, anticardiolipin antibody; APC, activated protein C; aPTT, activated partial thromboplastin time; AT III, antithrombin III; dRVVT, diluted Russel viper venom test; PT, prothrombin time; TIA, transient ischaemic attack. 
Vascular risk factors

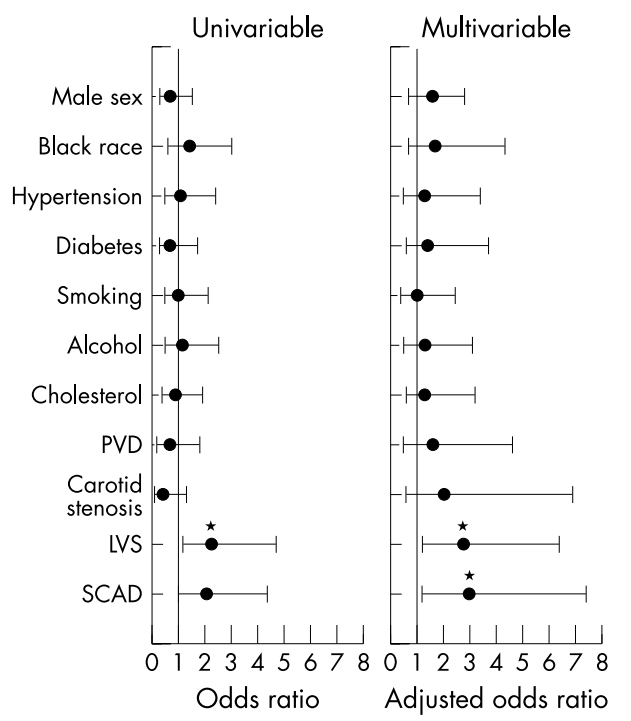

Figure 2 Results of univariate and multivariate analysis investigating the contribution of vascular risk factors to intracardiac thrombus formation in patients with recent strokes and transient ischaemic attacks.

atrial thrombus was found, suggesting that TOE may be superfluous in these patients. ${ }^{21}$ This discrepancy with the current study is probably a result of the systematic use of multiplanar TOE in a larger number of lacunar stroke patients $(n=50)$ and emphasises that patients presenting with lacunar infarction should not necessarily be excluded from TOE examination to rule out a cardiac source of embolism.

\section{Cardiological findings}

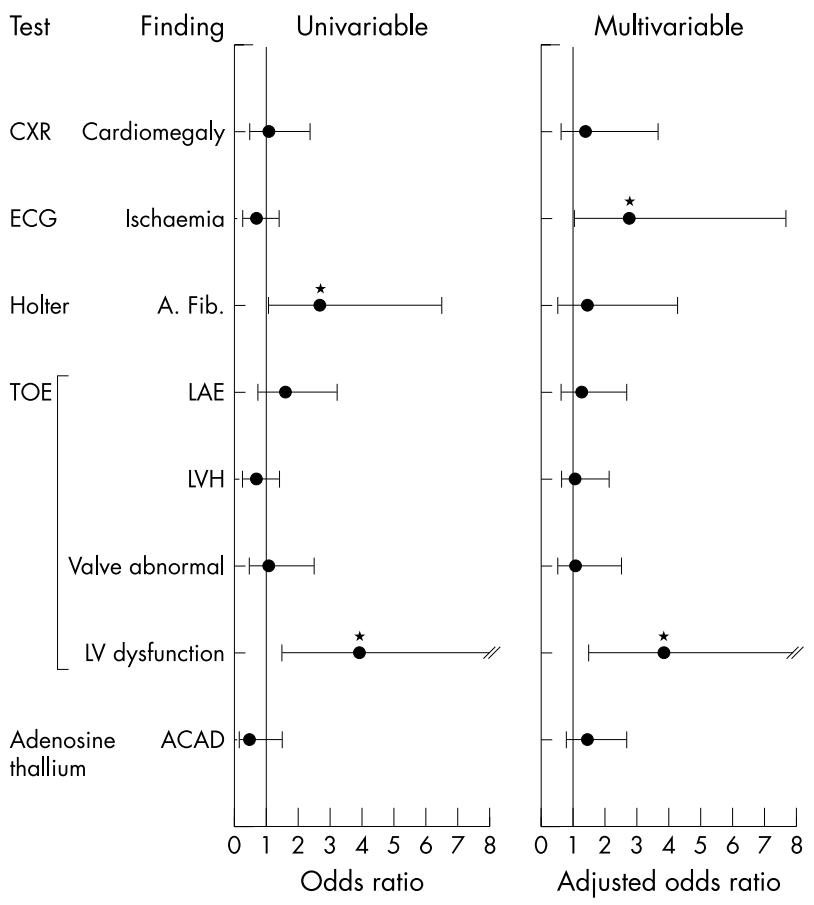

Figure 3 Results of univariate and multivariate analysis investigating the contribution of findings on routinely used cardiac tests to intracardiac thrombus formation in patients with recent strokes and transient ischaemic attacks.
TOE-determined left ventricular dysfunction was a significant risk factor for intracardiac thrombus on both univariate and multivariate analysis, in agreement with other studies. ${ }^{22}{ }^{23}$ Left ventricular dysfunction has been described as a predictor of left atrial appendage thrombus in non-stroke/TIA patients. ${ }^{24}$ As left ventricular function is better assessed by TTE than by TOE, ${ }^{25}$ TTE may be used to select those with left ventricular dysfunction, who may then undergo the more invasive examination to assess the presence of intracardiac thrombus.

Of the standard haematological studies, the total white blood cell count was higher in the thrombus group and only just escaped significance $(p=0.06)$; this may be related to a prothrombotic state associated with an occult infection. Alternatively it may represent an acute phase reaction to the stroke, which was larger in the thrombus group. ${ }^{26}$ However, other acute phase markers, such as ESR and the fibrinogen level, were not increased in patients with cerebral ischaemia who had intracardiac thrombus.

Indicators of a hypercoagulable state did not predict the presence of intracardiac thrombus, although a non-significant increase in protein $\mathrm{C}$ and antithrombin III deficiency was seen in these patients. As most haemorheological abnormalities are rare, the small number of patients is likely to have reduced the statistical power required for assessment of their contribution to the risk of intracardiac thrombus. Additionally, the rate at which these abnormalities were encountered in our study is unlikely to be related to an acute phase or inflammatory response as the ESR and fibrinogen values were similar in patients who had these coagulation deficiencies and those who did not. Further studies on a larger number of patients will be required to draw more definitive conclusions in this regard.

\section{Conclusions}

Coronary artery disease, large stroke on CT/MRI, atrial fibrillation, and ECG evidence of ischaemia are independent risk factors for intracardiac thrombus in patients with recent cerebral ischaemia. TOE evidence of left ventricular dysfunction is also a risk factor, though left ventricular dysfunction is best assessed by TTE. We suggest, therefore, that patients with recent cerebral ischaemia and the above factors, either alone or in combination, should be selected for TOE investigation, because of the $25 \%$ chance of intracardiac clot detection. As stroke recurrence is high in these patients if untreated, the use of these variables to focus TOE investigation on those with a high probability of harbouring an intracardiac thrombus may improve outcome and the risk/ benefit ratio.

\section{ACKNOWLEDGEMENTS}

Funding for the study was provided by the following USPHS (NIH) grants: PHS NS33770 and RR-00052

\section{Authors' affiliations}

S Laowatana, J Lima, S M Oppenheimer, Cerebrovascular Program, Departments of Neurology and Cardiology, The Johns Hopkins University School of Medicine, Baltimore, Maryland, USA

S Sen, UNC Stroke Program, Department of Neurology, University of North Carolina, Chapel Hill, North Carolina, USA

Competing interests: none declared

\section{REFERENCES}

1 Olsen TS, Skriver EB, Herning M. Cause of cerebral infarction in the carotid territory: its relation to the size and the location of the infarct and the underlying vascular lesion. Stroke 1985;16:459-6.

2 Bogousslavsky J, Melle GV, Regli F. The Lausanne Stroke Study: analysis of 1000 consecutive patients with first stroke. Stroke 1988;19:1083-92.

3 CE Task Force. Cardiogenic brain embolism. Arch Neurol 1989;46:727-43. 
4 Comess KA, DeRook FA, Beach KW, et al. Transesophageal echocardiography and carotid ultrasound in patients with cerebral ischemia: prevalence of findings and recurrent stroke risk. J Am Coll Cardiol 1994;23:1598-603.

5 Sen S, Lima JAC, Oppenheimer SM. 2004 Changes in cardiac thrombus status after cerebral ischemia. Cerebrovasc Dis 17:175-81.

6 Rauh G, Fischereder M, Spengel FA. Transesophageal echocardiography in patients with focal cerebral ischemia of unknown cause. Stroke 1996;27:691-4.

7 Albers GW, Comess KA, DeRook FA, et al. Transesophageal echocardiographic findings in stroke subtypes. Stroke 1994;25:23-8.

8 Manning WJ, Weintraub RM, Waksmonski CA, et al. Accuracy of transesophageal echocardiography for identifying left atrial thrombi. Ann Intern Med 1995;123:817-22.

9 Pearson AC, Labovitz AJ, Tatineni S, et al. Superiority of transesophageal echocardiography in detecting cardiac source of embolism in patients with cerebral ischemia of uncertain etiology. J Am Coll Cardiol 1991;17:66-72.

10 Lee RJ, Bartzokis T, Yeoh TK, et al. Enhanced detection of intracardiac sources of cerebral emboli by transesophageal echocardiography. Stroke 1991;22:734-9.

11 Cujec B, Polasek P, Voll C, et al. Transesophageal echocardiography in the detection of potential cardiac source of embolism in stroke patients. Stroke 1991;22:727-33.

12 Saperstein DS, Grometh GS. Cost-effectiveness analysis of echocardiography in stroke patients without cardiac risk factors [abstract]. Neurology 1998; 50:A446

13 Black IW, Hopkins AP, Lee LC, et al. Leff atrial spontaneous echo contrast: a clinical and echocardiographic analysis. J Am Coll Cardiol 1991;18:398-404.

14 Bamford J, Sandercock P, Dennis M, et al. Classification and natural history of clinically identifiable subtypes of cerebral infarction. Lancet 1991;337:1521-6.

15 Leung DY, Davidson PM, Cranney GB, et al. Thromboembolic risk of left atria thrombus detected by transesophageal echocardiogram. Am J Cardiol 1997;79:626-9.
16 Shively BK, Gelgand EA, Crawford MH. Regional left atrial stasis during atrial fibrillation. J Am Coll Cardiol 1996;27:1722-9.

17 Martin R, Bogousslavsky. Mechanism of late stroke after myocardial infarct: the Lausanne stroke registry. J Neurol Neurosurg Psychiatry 1993;56:760-4.

18 Funke Kupper AJ, Verheugt FWA, Peels $\mathrm{CH}$, et al. Left ventricular thrombus incidence and behavior studied by serial two-dimensional echocardiography in acute anterior wall myocardial infarction: left ventricular wall motion, systemic embolism and oral anticoagulation. J Am Coll Cardiol 1989;13:1514-20.

19 Nihoyannopoulos P, Smith GC, Maseri A, et al. The natural history of left ventricular thrombus in myocardial infarction: a rationale support of masterly inactivity. J Am Coll Cardiol 1989;14:903-11.

20 Sandercock P, Bamford J, Dennis $M$, et al. Atrial fibrillation and stroke: prevalence in different types of stroke and influence on early and long term prognosis (Oxfordshire Community Stroke Project). BMJ 1992;305:1460-5.

21 Censori B, Colombo F, Valsecchi MG, et al. Early transoesophageal echocardiography in cryptogenic and lacunar stroke and transient ischaemic attack. J Neurol Neurosurg Psychiatry 1998;64:624-7.

22 The Stroke Prevention in Atrial Fibrillation Investigators. Predictors of thromboembolism in atrial fibrillation: II. Echocardiographic features of patients at risk. Ann Intern Med 1992;116:6-12.

23 Archer SL, James KE, Kvernen LR, et al. Role of transesophagea echocardiography in the detection of left atrial thrombus in patients with chronic nonrheumatic atrial fibrillation. Am Heart J 1995; 130:287-95.

24 Brickner ME, Friedman DB, Cigarroa CG, et al. Relation of thrombus in the left atrial appendage by transesophageal echocardiography to clinical risk factors for thrombus formation. Am J Cardiol 1994;74:391-3.

25 Mugge A, Daniel WG, Haverich A, et al. Diagnosis of noninfective cardiac mass lesions by two-dimensional echocardiography. Comparison of the transthoracic and transesophageal approaches. Circulation 1991;83:70-8.

26 Syrianen J, Teppo AM, Valtonen VV, et al. Acute phase response in cerebral infarction. J Clin Pathol 1989;42:63-8. 